\title{
Measurements of the Isotopes of Lithium, Beryllium, and Boron from ACE/CRIS
}

\author{
G. A. de Nolfo*, N.E. Yanasak ${ }^{\dagger}$, W.R. Binns**, A.C. Cummings ${ }^{\dagger}$, E.R. Christian*, \\ J.S. George ${ }^{\dagger}$, P.L. Hink ${ }^{* *}$, M.H. Israel ${ }^{* *}$, R.A. Leske ${ }^{\dagger}$, M. Lijowski**, \\ R.A. Mewaldt ${ }^{\dagger}$, E.C. Stone ${ }^{\dagger}$, T.T. von Rosenvinge* and M.E. Wiedenbeck ${ }^{\ddagger}$ \\ ${ }^{*}$ Laboratory for High Energy Astrophysics, NASA/Goddard Space Flight Center, Greenbelt, MD, 20771 USA \\ 'Space Radiation Laboratory, California Institute of Technology, Pasadena, CA, 91125 USA \\ ${ }^{* *}$ Department of Physics and McDonnell Center for the Space Sciences, Washington University, St. Louis, MO, \\ 63130 USA \\ ${ }^{\ddagger}$ Jet Propulsion Laboratory, California Institute of Technology, Pasadena, CA, 91109 USA
}

\begin{abstract}
The isotopes of lithium, beryllium, and boron $(\mathrm{LiBeB})$ are known in nature to be produced primarily by CNO spallation and $\alpha-\alpha$ fusion from interactions between cosmic rays and interstellar nuclei. While the dominant source of $\mathrm{LiBeB}$ isotopes in the present epoch is cosmic-ray interactions, other sources are known to exist, including the production of ${ }^{7} \mathrm{Li}$ from big bang nucleosynthesis. Precise observations of galactic cosmic-ray $\mathrm{LiBeB}$ in addition to accurate modeling of cosmic-ray transport can help to constrain the relative importance among the different production mechanisms. The Cosmic Ray Isotope Spectrometer (CRIS) on the Advanced Composition Explorer (ACE) has measured nuclei with $2 \lesssim Z \lesssim 30$ in the energy range $\sim 30-500 \mathrm{MeV} /$ nucleon since 1997 with good statistical accuracy. We present measurements of the isotopic abundances of $\mathrm{LiBeB}$ and discuss these observations in the context of previous cosmic-ray measurements and spectroscopic observations.
\end{abstract}

\section{INTRODUCTION}

The elements lithium, beryllium, and boron ( $\mathrm{LiBeB})$ are rare in our galaxy and offer important constraints on our understanding of nuclear astrophysics. These fragile elements are not generated in appreciable amounts in stellar nucleosynthesis but are destroyed in stellar interiors, and this is reflected by their low abundance in nature (as measured in meteorites and stellar photospheres) [1]. Galactic cosmic-ray (GCR) $\mathrm{LiBeB}$ nuclei, on the other hand, are found to be in significant excess over their solar-system abundances. This excess is quite large ( $\mathrm{LiBeB} / \mathrm{CNO} \sim 0.25$ for GCRs compared with $\sim 10^{-6}$ for solar-system values) and is attributed to the spallation of CNO GCRs on the interstellar medium (ISM) [2]. A few isotopic discrepancies between GCR abundances and solar-system abundances, specifically the ratios of ${ }^{6} \mathrm{Li} /{ }^{7} \mathrm{Li}$ and ${ }^{11} \mathrm{~B} /{ }^{10} \mathrm{~B}$, are not consistent with spallation production, suggesting the need for other sources of the lithium and boron isotopes such as stellar nucleosynthesis [3] and neutrino spallation [4]. In addition, some ${ }^{7} \mathrm{Li}$ production is expected from primordial nucleosynthesis [5].

The evolution of $\mathrm{LiBeB}$ over the last $10 \mathrm{Gyr}$ has been assessed through observations of low-metallicity halo stars [6], [7]. These observations indicate an approximately linear correlation between the elemental ratios of $\mathrm{Be} / \mathrm{H}$ or $\mathrm{B} / \mathrm{H}$ and $\mathrm{Fe} / \mathrm{H}$ (or metallicity of the star), in contradiction with the expected quadratic behavior for the production of $\mathrm{LiBeB}$ nuclei from GCRs released into an ISM, which is itself increasing in metallicity with time from the contributions of evolving stars. A linear correlation suggests a primary origin of $\mathrm{LiBeB}$ nuclei not coupled to the ISM metallicity, such as the fragmentation products from freshly synthesized supernova ejecta. Several scenarios have been postulated to explain the observed correlation, including the origin of $\mathrm{LiBeB}$ species from within superbubble regions [8], [9], [10]. In this model, supernovae Type II (SN II), which are common in the early Galaxy and originate from stars in large associations, tend to sweep out a bubble filled with a warm, low density mixture of freshly synthesized supernovae ejecta and remaining interstellar material. Lowenergy carbon and oxygen $(<100 \mathrm{MeV} /$ nucleon $)$ are produced and accelerated within SN II, perhaps at the reverse shock [11],[12]. Once ejected, these low-energy carbon and oxygen nuclei are subsequently accelerated by massive stars or at the forward shocks of SN II in these

CP598, Solar and Galactic Composition, edited by R. F. Wimmer-Schweingruber

(C) 2001 American Institute of Physics 0-7354-0042-3/01/\$18.00 
superbubble regions and interact with interstellar hydrogen and helium to produce $\mathrm{LiBeB}$ abundances via fragmentation in agreement with observations of old Population II stars [13], [14]. Other models have been proposed, including the origin of $\mathrm{Be}$ and $\mathrm{B}$ nuclei by GCRs accelerated as debris from grains formed in SN II ejecta [15], [16].

Observations of GCR $\mathrm{LiBeB}$ nuclei help to constrain models of the origin and evolution of present epoch abundances. The Cosmic Ray Isotope Spectrometer (CRIS) on ACE has been measuring the isotopic composition of $2 \lesssim Z \lesssim 30$ in the energy range $\sim 30-500$ $\mathrm{MeV} /$ nucleon since 1997, including the abundances of LiBeB between 30-200 MeV/nucleon. CRIS observations of $\mathrm{LiBeB}$ nuclei originate almost exclusively from fast CNO GCRs interacting with the ISM to produce secondary LiBeB nuclei between 200-500 MeV/nucleon. The resulting GCR $\mathrm{LiBeB}$ nuclei depend on the fragmentation rate and the rate of escape from the Galaxy. Indeed, most of the GCR LiBeB escape from the Galaxy. As the escape rate is essentially the same for all $\mathrm{LiBeB}$ species of equal energy per nucleon [17], the relative abundances are a direct measure of the relative production rates. Approximately half of the $\mathrm{LiBeB}$ nuclei produced by GCRs, however, result from the fragmentation of ISM by GCR protons and helium whose energy spectra peak around $1 \mathrm{GeV}$. Provided the cross-sections are not too different between the interactions of ISM (p,He) $+\mathrm{GCR}(\mathrm{CNO})->\mathrm{LiBeB}$ around $1 \mathrm{GeV}$ and the inverse interactions of ISM $(\mathrm{CNO})+\mathrm{GCR}(\mathrm{p}, \mathrm{He})->\mathrm{LiBeB}$ in the energy range covered by CRIS, the observed abundances of $\mathrm{LiBeB}$ isotopic ratios from CRIS should reflect the actual isotopic ratios observed in the ISM. Indeed, based on a review of the cross-sections of Read and Viola [18] by Yanasak et al. (these proceedings), the cross-sections between 200-500 MeV/nucleon and those between 500-2000 MeV/nucleon for the inverse reactions are on average less than $20 \%$ different. The ISM ratio ${ }^{6} \mathrm{Li} /{ }^{7} \mathrm{Li}$, however, may not be similar to the GCR ratio since the cross-section for $\alpha+\alpha$ fusion to $A=6,7$ increases by more than 2 orders of magnitude between 10 and $100 \mathrm{MeV} /$ nucleon, implying that a large fraction of ISM lithium may be produced at these low energies. Thus, GCR LiBeB observations over a wide range in energy can provide important constraints on cosmicray origin and evolution in addition to deciphering the dominant sources of $\mathrm{LiBeB}$ over time. In this paper, we present the measurements of GCR LiBeB from December 1997 through April 2000 and compare these observations with previously published results.

\section{DATA ANALYSIS}

CRIS identifies the charge and mass of incident particles using multiple $\mathrm{dE} / \mathrm{dx}$ measurements and the total energy deposited within stacked silicon solid-state detectors [19]. The mass resolution is refined with the measurement of particle trajectory via a scintillating optical fiber hodoscope (SOFT) [19]. In the present study, CRIS observations of $\mathrm{LiBeB}$ are separated into two separate time intervals, corresponding to differing levels of solar modulation. The first time period ranges from December 20, 1997 to January 23, 1999, which corresponds to a total of 311 days, excluding periods of intense solar activity. The second period ranges from January 24, 1999 to April 18, 2000 corresponding to a total of 270 days, again excluding periods of intense solar activity. The effect of solar modulation experienced during these two time intervals is determined using a spherically symmetric model described by Fisk [20]. The solar modulation parameter, $\phi$, is determined by fitting elemental spectra from CRIS at low energies and from HEAO-3 at high energies with the predictions of a propagation model [21]. The first interval corresponds to $\phi \sim 400 \mathrm{MV}$ and the second time interval corresponds to $\phi \sim 590 \mathrm{MV}$.

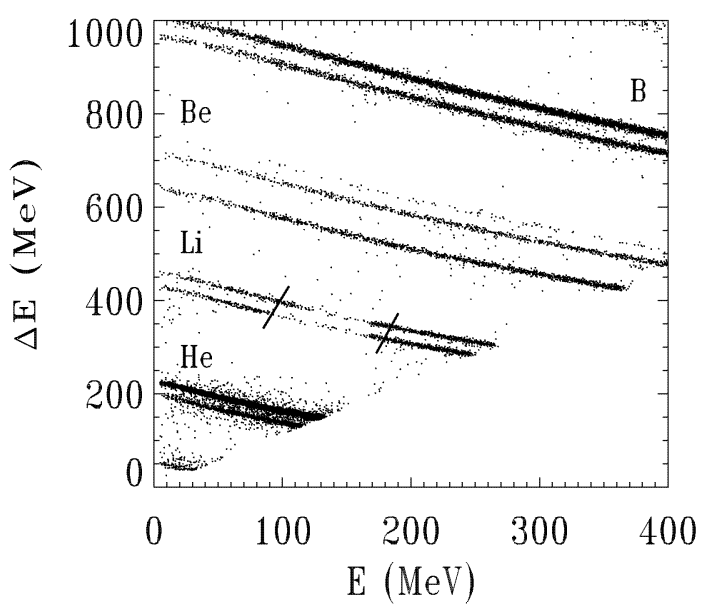

FIGURE 1. Energy loss ( $\Delta \mathrm{E})$ versus the energy $(\mathrm{E})$ deposited in a detector element midway through the stack for $\mathrm{He}, \mathrm{Li}, \mathrm{Be}$, and $\mathrm{B}$ events. The solid lines indicate the region excluded from the analysis to exclude events within the low duty-cycle helium event buffer.

CRIS is designed to transmit only a small sample of the highly abundant helium events [19]. Since the onboard classification of an event as helium is rather coarse and based only on pulse heights, and since lithium is adjacent to helium, lithium events within certain energy intervals are treated as helium and sampled at a reduced duty cycle. Figure 1 shows an example of the energy loss versus the energy deposited in two detectors mid- 

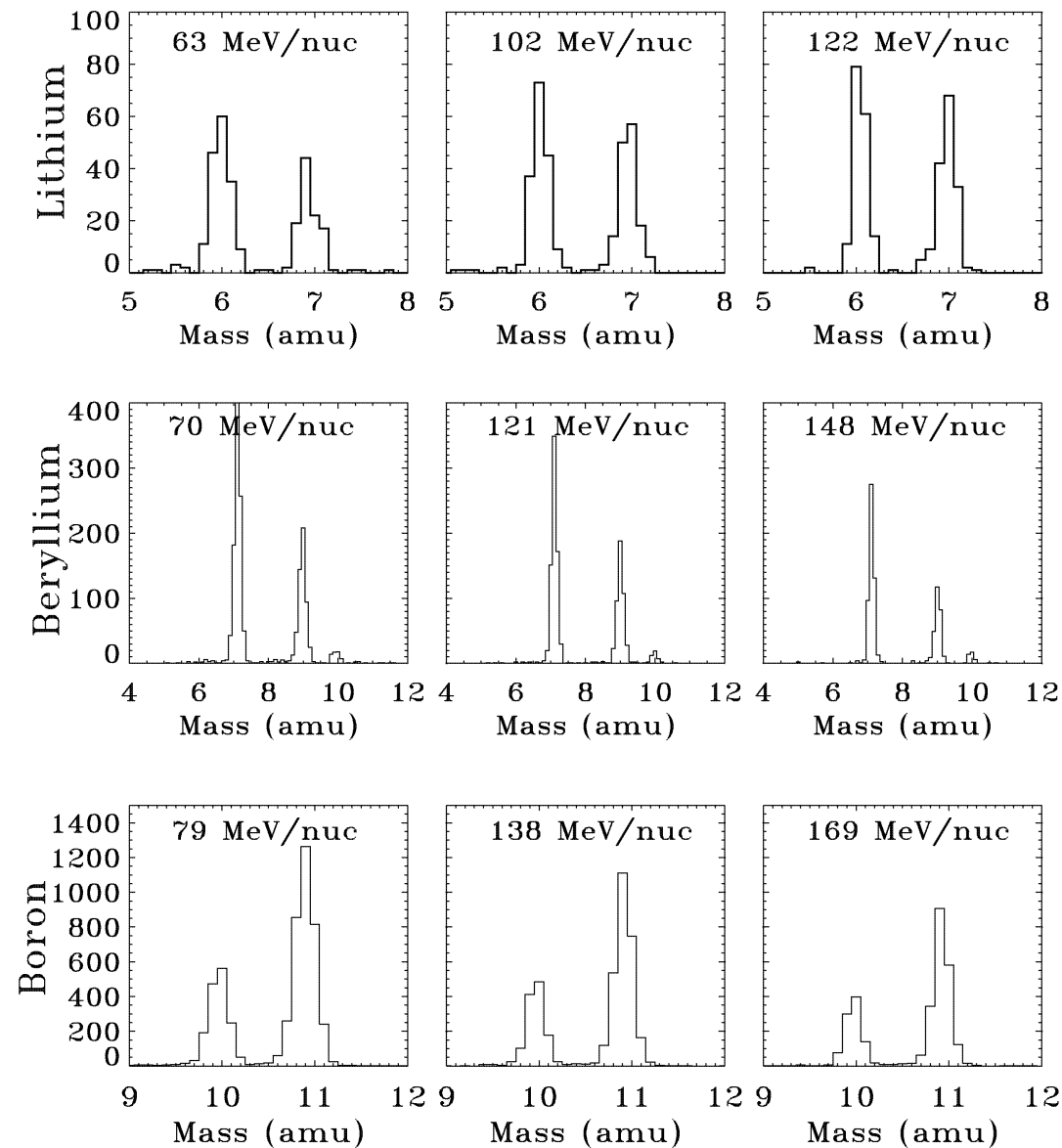

FIGURE 2. Mass histograms for the isotopes of $\mathrm{LiBeB}$ in three of the six energy ranges covered in this study. The labels refer to the median energy for each range.

way through the CRIS stack for events with $Z<6$. From Figure 1 , it is clear that CRIS obtains excellent mass resolution. The solid lines indicate a region where lithium events have been classified into a helium event buffer which is sampled at a lower rate, resulting in a lower density region in the track. In the present analysis, events located within an interval such as that shown in Figure 1 are excluded, ensuring that those events with pulse heights satisfying the helium event buffer are eliminated from the analysis.

Particles are selected on the basis of high-quality mass and charge identification. Valid events are required to trigger all three SOFT hodoscope planes. Furthermore, events that stop near the faces of the detector elements are rejected from the analysis. Additional background events are removed by demanding consistency between the measures of charge and mass from various combinations of the stack detector pulse heights [19].

The resulting mass histograms for $\mathrm{LiBeB}$ events in the first time period are shown in Figure 2 for three of the six energy bins analyzed in this study, ranging from $\sim 30$ $\mathrm{MeV} /$ nucleon to $\sim 200 \mathrm{MeV} /$ nucleon. The isotopes of $\mathrm{LiBeB}$ are clearly separated and the number of events for each isotope is simply determined by adding the number of events under each peak. Only events with an opening angle from the detector zenith of $<25$ degrees are included in the analysis. Corrections are applied to account for the probability of a particle surviving fragmentation within the instrument and for the charge dependent tracking efficiency with the SOFT hodoscope. The spallation correction accounts for the number of interaction lengths penetrated and is based on the cross-section formula of Westfall et al. [22].

\section{RESULTS AND DISCUSSION}

Figure 3 shows the elemental ratio of $\mathrm{B} / \mathrm{C}$ and the isotopic ratios of ${ }^{6} \mathrm{Li} /{ }^{7} \mathrm{Li},{ }^{7} \mathrm{Be} /{ }^{9} \mathrm{Be}$, and ${ }^{11} \mathrm{~B} /{ }^{10} \mathrm{~B}$ for the first time period along with previous measurements of these 

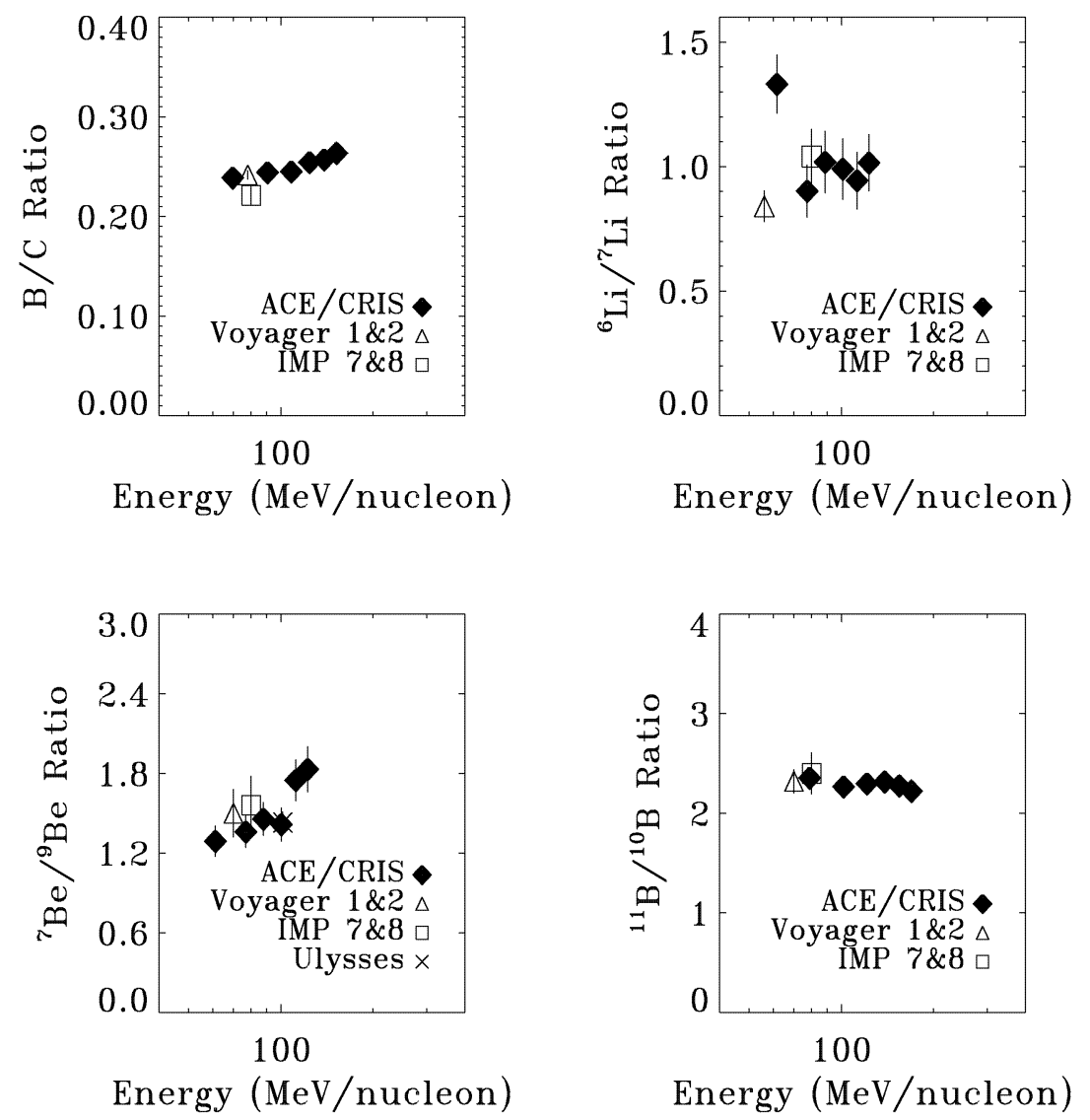

FIGURE 3. The ratios of ${ }^{6} \mathrm{Li} /{ }^{7} \mathrm{Li},{ }^{6} \mathrm{Li} / \mathrm{C},{ }^{7} \mathrm{Li} / \mathrm{C}$ and $\mathrm{Li} / \mathrm{C}$ obtained from CRIS during the first time interval compared with previous measurements. The observations of IMP $7 \& 8$ and Voyager $1 \& 2$ were obtained during comparable periods of solar modulation.

ratios from Voyager $1 \& 2$ [23] and IMP $7 \& 8$ [24] and Ulysses [25]. Observations from Voyager $1 \& 2$ were made over 19 years at an average solar modulation level of $\phi \sim 450 \mathrm{MV}$ and IMP $7 \& 8$ observations were made at a solar modulation level of $\phi \sim 430 \mathrm{MV}$. These measurements were obtained during periods of comparable solar modulation to that experienced by CRIS during the first time interval. CRIS observations of $\mathrm{B} / \mathrm{C}$ and the isotopic ratios of ${ }^{6} \mathrm{Li} /{ }^{7} \mathrm{Li},{ }^{7} \mathrm{Be} /{ }^{9} \mathrm{Be}$, and ${ }^{11} \mathrm{~B} /{ }^{10} \mathrm{~B}$ are in agreement with both Voyager and IMP to within the statistical accuracy of the measurements. Indeed, CRIS observations are unique in that there are now sufficient events to measure these ratios as a function of energy, providing further constraints on cosmic-ray propagation models.

Table 1 compares the isotopic measurements of $\mathrm{LiBeB}$ with previous measurements, where the results have been averaged over the entire energy range covered by CRIS. The uncertainties for CRIS in Table 1 represent only the statistical uncertainty. Typical systematic uncertainties are $\sim 10 \%$ for beryllium and lithium due to the SOFT tracking efficiency. The predictions of two propagation models from Reeves [3] and Lukasiak et al. [23] are also shown in Table 1. While CRIS results agree with previous measurements and with the predictions of the propagation model by Lukasiak et al. [23], the measured isotopic ratios differ, not unexpectedly, with the calculations of Reeves [3] for GCRs in the several $\mathrm{GeV} /$ nucleon energy range. The effect of energy dependent cross-sections on the production of GCR $\mathrm{LiBeB}$ nuclei and the expected energy dependence of the $\mathrm{LiBeB}$ ratios compared with these observations will be explored in detail in a companion paper in these proceedings (Yanasak et al.) [26].

Comparing the relatively young GCRs $\left(\sim 1.5 \times 10^{7}\right.$ years [27]) with presolar isotopic abundances can shed light on the evolution of $\mathrm{LiBeB}$ in general. The ratio of ${ }^{7} \mathrm{Li} /{ }^{6} \mathrm{Li}$ is well below the solar-system value of $\sim 12 \mathrm{de}-$ termined from meteorites [1]. The ${ }^{11} \mathrm{~B} /{ }^{10} \mathrm{~B}$ ratio is also well below the ratios determined from meteorites (4.05 $\pm 0.2)$ [28] and from present abundance measurements 
TABLE 1. LiBeB ratios compared with other observations and with the Leaky Box Model (LBM) prediction of Lukasiak et al. 1999 [23] and of Reeves et al. 1994 [3]. The solar modulation for CRIS in period I and II are $\phi \sim 400 \mathrm{MV}$ and $590 \mathrm{MV}$, respectively. Uncertainties are statistical only.

\begin{tabular}{|c|c|c|c|c|c|c|}
\hline Ratios & $\begin{array}{c}\text { CRIS I } \\
(40-130) \\
\text { (MeV/nucleon) }\end{array}$ & $\begin{array}{c}\text { CRIS II } \\
(40-130) \\
(\mathrm{MeV} / \text { nucleon })\end{array}$ & $\begin{array}{c}\text { IMP 7\&8 } \\
(31-151) \\
\text { ( MeV/nucleon) }\end{array}$ & $\begin{array}{c}\text { Voyager } 1 \& 2 \\
\sim 80 \\
\text { (MeV/nucleon) }\end{array}$ & $\begin{array}{c}\text { LMB (1999) } \\
\sim 100 \\
\text { (MeV/nucleon) }\end{array}$ & $\begin{array}{c}\text { Reeves (1994) } \\
\sim 1 \\
\text { (GeV/nucleon) }\end{array}$ \\
\hline${ }^{7} \mathrm{Li} /{ }^{6} \mathrm{Li}$ & $0.97 \pm 0.05$ & $1.12 \pm 0.08$ & $0.97 \pm 0.11$ & $1.19 \pm 0.09$ & 1.26 & 1.4 \\
\hline${ }^{7} \mathrm{Be} /{ }^{9} \mathrm{Be}$ & $1.54 \pm 0.06$ & $1.41 \pm 0.07$ & $1.56 \pm 0.12$ & $1.50 \pm 0.18$ & 1.43 & - \\
\hline${ }^{11} \mathrm{~B} /{ }^{10} \mathrm{~B}$ & $2.29 \pm 0.04$ & $2.36 \pm 0.04$ & $2.40 \pm 0.21$ & $2.32 \pm 0.12$ & 2.49 & 2.5 \\
\hline $\mathrm{B} / \mathrm{C}$ & $0.250 \pm 0.004$ & $0.265 \pm 0.004$ & $0.220 \pm 0.009$ & $0.240 \pm 0.005$ & 0.237 & - \\
\hline
\end{tabular}

of the ISM $(3.4 \pm 0.7)[29]$. In fact, the meteoritic observations of ${ }^{7} \mathrm{Li} / 6 \mathrm{Li}$ and ${ }^{11} \mathrm{~B} /{ }^{10} \mathrm{~B}$, representing protosolar measurements, are consistent with measurements of Population I stars (see compilation from [30] and [31]). The difference between the $\mathrm{GCR}{ }^{7} \mathrm{Li} /{ }^{6} \mathrm{Li}$ and the ${ }^{11} \mathrm{~B} /{ }^{10} \mathrm{~B}$ ratios and the solar-system values has been attributed to excess ${ }^{7} \mathrm{Li}$ and ${ }^{11} \mathrm{~B}$, postulated to originate in part from neutrino spallation in core collapse supernovae [16]. The isotope ${ }^{7} \mathrm{Li}$ is also produced during primordial nucleosynthesis $(\sim 1 / 10$ solar $)$ [32]. Furthermore, halo stars with even lower metallicities $[\mathrm{Fe} / \mathrm{H}] \sim-2.3$ indicate a ${ }^{6} \mathrm{Li} /{ }^{7} \mathrm{Li}$ ratio of 0.05 [31]. While GCRs play a dominant role in the production of LiBeB nuclei, the origin and evolution of these species is complex as is reflected in the variety of observations from different epochs over the last $10 \mathrm{Gyr}$.

\section{SUMMARY}

The isotopic ratios of $\mathrm{LiBeB}$ and $\mathrm{C}$ are in agreement with previous measurements of GCRs. The observed ratios during both time periods are also in agreement suggesting that the effects of solar modulation are not significant, though this will be tested further with future observations from CRIS and the Solar Isotope Experiment (SIS) on-board ACE during periods of extreme solar modulation. While current propagation models are in general agreement with the LiBeB observations, CRIS observations over an extended energy range will provide further constraints on propagation models as will be discussed in the companion paper (Yanasak et al.) [26].

\section{ACKNOWLEDGMENTS}

This work was supported by NASA at NASA/Goddard Space Flight Center, the Jet Propulsion Laboratory, Washington University and the California Institute of Technology (under grant NAG5-6912).

\section{REFERENCES}

1. Anders, E., and Grevesse, N., Geochim. Cosmochim. Acta, 53, 197 (1989).

2. Reeves, H., et al., Nature, 226, 727 (1970).

3. Reeves, H., Rev. Mod. Phys., 66, 193 (1994).

4. Woosley, S., and Weaver, T., ApJ Suppl., 101, 181 (1995).

5. Schramm, D., Origin and Evolution of the Elements, N. Prantzos et al., Cambridge University, Cambridge, England, 1993, p. 112.

6. Ryan, S., et al., ApJ, 388, 184 (1994).

7. Duncan, D., et al., ApJ, 488, 338 (1997).

8. Bykov, A., "LiBeB", in LiBeB Cosmic Rays and GammaRay Line Astronomy, edited by R. Ramaty et al., ASP Conference Series, 1999, vol. 171, p. 146.

9. Parizot, E., et al., Astron. Astrophys., 328, 107 (1997).

10. Higdon, H., et al., Proc. $26^{\text {th }}$ ICRC, 4, 144 (1999).

11. Parizot, E., and Drury, L., Astron. Astrophys., 346, 329 (1999).

12. Ramaty, R., et al., ApJ, 488, 730 (1997).

13. Cásse, M., et al., Nature, 373, 318 (1995).

14. Ellison, D., et al., ApJ, 487, 197 (1997).

15. Ryan, S., et al., ApJ, 488, 730 (1997).

16. Vangioni-Flam, E., et al., ApJ, 468, 199 (1996).

17. Garcia-Munoz, M., et al., ApJ Suppl., 64, 269 (1987).

18. Read, S., and Viola, V., Atomic Data Nucl. Data Tables, 31, 359 (1984).

19. Stone, E., et al., Space Sci. Rev., 86, 285 (1998).

20. Fisk, L., JGR, 76, 221 (1971).

21. Davis, A., Phys. Rev. C, 528, 421 (2000).

22. Westfall, G., Phys. Rev. C, 19, 1309 (1979).

23. Lukasiak, A., et al., Proc. $26^{\text {th }}$ ICRC, 3, 41 (1999).

24. Garcia-Munoz, M., et al., Proc. $17^{\text {th }}$ ICRC, 2, 72 (1981).

25. Connell, J., ApJ, 501, L59 (1998).

26. Yanasak, N., et al., these proceedings (2001).

27. Yanasak, N., et al., Proc. $26^{\text {th }}$ ICRC, 3, 9 (1999).

28. Chaussidon, M., and Robert, F., Nature, 374, 337 (1995).

29. Lambert, D., et al., ApJ, 494, 614 (1998).

30. Lemoine, M., et al., ApJ, 499, 735 (1998).

31. Hobbs, L., Physics Reports, 333-334, 449 (2000).

32. Spite, F., and Spite, M., Origin and Evolution of the Elements, N. Prantzos et al., Cambridge University, Cambridge, England, 1993, p. 201. 